\title{
Parents' Readiness to Change Affects BMI Reduction Outcomes in Adolescents with Polycystic Ovary Syndrome
}

\author{
Karen P. Jakubowski, ${ }^{1}$ Jessica J. Black, ${ }^{2}$ Nermeen E. El Nokali, ${ }^{3}$ Katherine A. Belendiuk, ${ }^{4}$ \\ Tamara S. Hannon, ${ }^{5}$ Silva A. Arslanian, ${ }^{3}$ and Dana L. Rofey ${ }^{1,3}$ \\ ${ }^{1}$ Department of Psychiatry, University of Pittsburgh School of Medicine, Pittsburgh, PA 15260, USA \\ ${ }^{2}$ Department of Psychology, University of Cincinnati, Cincinnati, OH 45221, USA \\ ${ }^{3}$ Division of Weight Management and Wellness, Department of Pediatrics, University of Pittsburgh School of Medicine, \\ Pittsburgh, PA 15224, USA \\ ${ }^{4}$ Department of Psychology, University of Pittsburgh, Pittsburgh, PA 15260, USA \\ ${ }^{5}$ Department of Pediatrics, Indiana University School of Medicine, Indianapolis, IN 46202, USA
}

Correspondence should be addressed to Dana L. Rofey, rofeydl@upmc.edu

Received 5 December 2011; Revised 23 April 2012; Accepted 6 June 2012

Academic Editor: Pedro J. Teixeira

Copyright ( $) 2012$ Karen P. Jakubowski et al. This is an open access article distributed under the Creative Commons Attribution License, which permits unrestricted use, distribution, and reproduction in any medium, provided the original work is properly cited.

\begin{abstract}
Evidence supports the importance of parental involvement for youth's ability to manage weight. This study utilized the stages of change (SOC) model to assess readiness to change weight control behaviors as well as the predictive value of SOC in determining BMI outcomes in forty adolescent-parent dyads (mean adolescent age $=15 \pm 1.84$ (13-20), BMI $=37 \pm 8.60 ; 70 \%$ white) participating in a weight management intervention for adolescent females with polycystic ovary syndrome (PCOS). Adolescents and parents completed a questionnaire assessing their SOC for the following four weight control domains: increasing dietary portion control, increasing fruit and vegetable consumption, decreasing dietary fat, and increasing usual physical activity. Linear regression analyses indicated that adolescent change in total SOC from baseline to treatment completion was not predictive of adolescent change in BMI from baseline to treatment completion. However, parent change in total SOC from baseline to treatment completion was predictive of adolescent change in BMI, $(t(24)=2.15, p=0.043)$. Findings support future research which carefully assesses adolescent and parent SOC and potentially develops interventions targeting adolescent and parental readiness to adopt healthy lifestyle goals.
\end{abstract}

\section{Introduction}

Obesity is the most common chronic health condition in pediatrics. In the United States, approximately 17\% (or 12.5 million) of children and adolescents aged 2-19 years are obese (body mass index $\geq 95$ th percentile), and the prevalence of obesity has nearly tripled since 1980 [1]. The necessity of effective prevention and treatment programs cannot be overstated, particularly when considering the severity and expense of obesity-related medical conditions in children and adolescents (e.g., diabetes mellitus, asthma, and high blood pressure). Polycystic ovary syndrome (PCOS) is a common, chronic condition often associated with obesity that affects approximately $6-10 \%$ of women of reproductive age [2]. PCOS is characterized by anovulation, hyperandrogenism, and insulin resistance, and approximately $70-74 \%$ of adolescents with PCOS are obese [3]. It is increasingly being recognized in adolescent girls who seek treatment for symptoms of hyperandrogenism [4].

Lifestyle modification encouraging dietary and physical activity change is the standard treatment for overweight and obese youth $[5,6]$. Consequently, readiness to change eating and activity behaviors likely impacts obesity treatment outcomes. This may be particularly important for adolescents with PCOS, as the condition is associated with depression in nearly half of the diagnosed cases, and this may impact readiness to make behavioral change [7-11]; thus it is important to understand how to help patients make effective lifestyle changes.

The transtheoretical or Stages of Change (SOC) model describes individuals' readiness to make behavioral changes 
[12]. In this model, the process of change is explained as a series of stages. These stages of change, as defined by DiClemente and Prochaska [12], are precontemplation (unaware of problem and without intention to change), contemplation (aware of problem and with intention to change), preparation (planning for change), action (implementing behavior change for less than six months), and maintenance (implementing behavior change for more than six months). The stages of change describe change as an unfolding process, one that is fluid and does not necessarily move in a linear fashion from one stage to the next.

There is support for considering SOC in adult weight management. First, SOC has been shown to capture current weight-related behaviors such as fat intake, effectively characterizing adults into pre-action (precontemplation or contemplation) or action stages according to participants' self-reported current dietary fat intake and confidence in their ability to make changes to their dietary fat intake in the future [13]. SOC also has substantial support as a predictor of positive lifestyle changes in adults, such as predicting readiness to make broad health changes learned in a diabetes educational program [14], as well as improved nutrition $[15,16]$. More specifically, results from Glanz and colleagues [15] indicate that SOC was a significant predictor of dietary habits for adults, explaining $93 \%$ of the variance in dietary intake for fat and $73 \%$ of the variance for fruits and vegetables, even after controlling for important covariates such as BMI and demographics. Additionally, Greene and colleagues [16] found a positive association between SOC and fruit and vegetable intake, particularly a linear increase in intake from individuals in the precontemplation stage to individuals in action/maintenance stages. SOC has also been used to predict level of physical activity in adults, including minutes of moderate to very hard intensity, as well as increased physical activity based on stage-tailored physical activity interventions [17-21].

While the literature involving interventions in adolescents is considerably less robust than that for adults, there is a growing body of work describing promising interventions for adolescents for a range of health-related behaviors including smoking cessation [22], weight control [6, 2326], and metabolic control in adolescents with diabetes [27]. Furthermore, there are a growing number of studies assessing SOC in adolescents. The aforementioned paragraph demonstrated that SOC predicts dietary and physical activity habits in adults, yet evidence shows that readiness to change may also be predictive of success in interventions targeting the improvement of glycemic control in adolescents with diabetes [28], lowering dietary fat intake in adolescents [29], and decreasing snack/dessert consumption in overweight and obese adolescents [30].

Despite the evidence that SOC may help predict outcomes in adolescents' readiness to make healthy lifestyle changes, these changes do not happen in isolation. Parents' dietary and physical activity SOC may also be influential for their adolescents' behaviors. Research indicates that family eating and lifestyle habits may provide an "obesogenic" environment for children and adolescents [31]. It has been shown that children who are more physically active are more likely to have physically active parents [32] and that the fruit and vegetable intake of young girls is positively related to parental fruit and vegetable intake [33]. Parents typically play a critical role in the home food environment as they are often the ones purchasing food and preparing meals. Furthermore, parental readiness to change eating behaviors is potentially important to adolescent outcomes because adolescents may adopt behaviors modeled by their parents [34]. Conversely, adolescents may be limited in their healthy food choices within the home given the type of food provided by parents. Thus, adolescents who are ready to make positive changes to their lifestyle choices may be stalled by parental SOC. However, little work has been conducted on the relationship between parent and adolescent SOC for weight management. Thus there is a need to fill this gap in the literature.

In addition to the potential influence of parent practices on adolescent SOC, there are a number of other factors that may impact adolescent readiness to change weightrelated lifestyle choices. Co-occurring medical conditions such as type 2 diabetes and asthma may be associated with a higher SOC for adolescents, as positive weight management practices improve physical illness symptoms. On the other hand, common comorbid psychiatric disorders such as depression may decrease adolescents' motivation to make healthy changes $[35,36]$. Given that this sample consists of adolescents with PCOS (a possible positive medical motivator) and depressive symptoms (a possible negative marker), it allows for the opportunity to elucidate the importance of SOC in a challenging, yet real world sample. The presence of a positive medical motivator like PCOS may also be an influential factor for mothers, who made up the majority of parent participants in the present study and who may have had PCOS themselves. Given these issues, the current study allows us to descriptively examine how simultaneous medical and psychiatric diagnoses may influence adolescents' SOC.

Taken together, the evidence reviewed suggests the importance of considering the impact of both parent and adolescent SOC on adolescents' abilities to make weightrelated changes. Although family-based treatment programs have demonstrated success in the pediatric obesity literature, and supporting evidence indicates that parents' commitment to weight management is the best predictor of children's weight management success [37], there is little evidence with regards to the relationship between parents' readiness to change weight control behaviors and weight loss in their adolescent children. Given the strong support for family-based treatment programs, but the relative paucity of information on the utility of parent and adolescent SOC in weight management interventions, it is important to fill this gap in the literature. In this study we aimed to: (1) examine SOC in adolescents and parents participating in a pediatric weight management intervention at baseline and treatment completion, and (2A) examine associations between both adolescent and parent total baseline SOC and adolescent change in BMI at study completion, as well as (2B) examine associations between both adolescent and parent change in total SOC from baseline to treatment completion and change in adolescent BMI at treatment completion. Specifically, we 
hypothesize that (1) adolescent baseline total SOC scores will predict adolescent change in BMI from baseline to treatment completion (six months) and (2) parent baseline total SOC scores will be a stronger predictor of adolescent change in BMI from baseline to treatment completion (six months). Additionally, since the stages of change are described as an unfolding, fluid process, one that may be influenced by motivational interviewing techniques, it is possible that baseline total SOC is not correlated with SOC across the course of treatment. Thus, exploratory analyses were conducted to measure associations between changes in adolescent BMI and changes in total SOC from baseline to treatment completion for both adolescents and parents.

\section{Methods}

Data for this investigation came from a larger 11-session family-based intervention employing Cognitive Behavioral Therapy (CBT) and Motivational Interviewing (MI) to target weight management and mood improvement in obese adolescents with polycystic ovary syndrome (PCOS) and comorbid depression (K12-HD043441 and K23HD061598 (DLR)). The larger study was approved by the University of Pittsburgh Institutional Review Board.

2.1. Participants. This study analyzed data from forty adolescent-parent dyads who agreed to participate in a psychosocial treatment study for adolescents with PCOS. The majority of adolescents in this sample were obese as defined by the Centers for Disease Control and Prevention criteria (BMI $\geq 95$ th percentile, for children of the same age and sex [37]), while three cases were categorized as overweight (95th percentile $>$ BMI $\geq 85$ th percentile, for children of the same age and sex [37]). These adolescents were Englishspeaking females ages 13-20 (mean age $=15 \pm 1.84$ [12-19], $\mathrm{BMI}=37 \pm 8.60 ; 70 \%$ white, $22 \%$ African American, $8 \%$ biracial) primarily recruited for treatment at the Polycystic Ovary Syndrome Center or Adolescent Medicine Clinic at an urban Mid-Atlantic Children's Hospital. All participants had a confirmed diagnosis of PCOS based on clinical and laboratory evidence of hyperandrogenism and co-occurring, clinically significant depressive symptoms per a semistructured interview (K-SADS-PL [38]; see Measures). After receiving a comprehensive explanation of the study protocol and course of treatment, participants and/or their parents signed informed consent. The intervention was provided free of charge, and participants were compensated for completion of study measures; see Table 1 for inclusion and exclusion criteria.

2.2. Procedures. The intervention consisted of one-on-one sessions between adolescents and study interventionists, who were Masters- and Ph.D.-level clinicians trained as therapists, dieticians, and/or exercise physiologists. Sessions consisted primarily of CBT and MI for four weekly sessions, four biweekly sessions, and three monthly booster sessions (see Table 2 for a description of sessions). An engagement session, which occurred at the first meeting with each participant, allowed for presentation of the key principles of MI: suspension of the clinician's assumptions, utilizing open-ended questions, expression of empathy and reflective listening, working with patients' resistance, discussing change and adherence, and supporting the patients' self-efficacy [39]. This engagement session served the purpose to enhance the likelihood that adolescents with PCOS would enter, attend, and participate actively in the CBT protocol. For the purposes of this intervention, CBT and MI served as complementary approaches: CBT served as the empiricallyvalidated therapeutic approach, teaching adolescents to reduce their physical and emotional disturbances, while MI was used to elicit health behavior change by enhancing intrinsic motivation [40]. Given MI's emphasis on increasing internal motivation for change, the techniques of MI may allow clinicians to assess the degree of motivation associated with individuals' readiness to make and maintain behavioral changes (albeit not synonymous with SOC) [41]. This is important in the context of SOC, as MI may help elucidate underlying aspects of readiness to change that lead to one's initial SOC and, perhaps more importantly, that stimulate subsequent movements between SOC throughout the course of treatment; for example, wanting to look more attractive or wanting to learn and implement strategies to improve diet and exercise [41]. Thus the tenets of MI are likely very useful in assessing and promoting specific areas of readiness to change that may lead to the evolution of SOC in individuals seeking treatment in a weight management program.

The eleven sessions, roughly an hour each, were intended to concurrently address mood and weight management in order to address the bidirectional relationship between managing weight and managing emotions. The overarching aim of the program was to encourage participants to increase their physical activity and make better nutritional choices in an effort to reduce weight as well as obtain skills to help them manage their emotions. Overall, the program employed a family-based approach, and the participation of at least one invested parent/guardian was strongly recommended for all participants, even those over 18 years of age. In addition to parent/guardian participation at the end of each session, during adolescent sessions one, four, and eight, parents were given the opportunity to participate in 30-minute sessions (with or without their child present) with the study interventionist. These three familybased sessions corresponded to the adolescent sessions and provided suggestions for how parents could support and encourage their adolescents' weight-related changes. The sessions were particularly focused on (1) creating a more optimal home environment for weight loss and increased physical activity (e.g., stimulus control, healthy cooking at home, and healthy eating when dining out), (2) facilitating more effective parenting using behavioral strategies (e.g., increasing praise/reinforcement and setting limits and rules to help adolescents make positive weight-related changes), and (3) summarizing the program and looking ahead (e.g., discussing strategies for nutrition and physical activity that could assist lifestyle changes, such as logging daily activity and foods eaten). Pilot data for the intervention indicated a significant reduction in weight, $t(11)=6.6, p<.05$ with 
TABLE 1: Inclusion and exclusion criteria.

\begin{tabular}{|c|c|c|}
\hline Inclusion criteria & \multicolumn{2}{|c|}{$\begin{array}{l}\text { (1) Confirmed PCOS diagnosis by a board-certified physician } \\
\text { (2) BMI percentile }<85 \\
\text { (3) CDI or CDI-P }<10 \\
\text { (4) DSM-IV diagnosis of minor or major depressive disorder as reported on the K-SADS-PL } \\
\text { (5) Presence of at least one parent or guardian if } \leq 18 \text { years of age } \\
\text { (6) Age between } 11 \text { and } 21 \text {, inclusive }\end{array}$} \\
\hline Exclusion criteria & \multicolumn{2}{|c|}{$\begin{array}{l}\text { (1) History or current episode of bipolar disorder or psychotic disorder by DSM-IV criteria } \\
\text { (2) Suicidality with plan or of severity requiring immediate psychiatric hospitalization or significant act } \\
\text { involving intentional self-harm (e.g., cutting or overdose, resulting in medical attention) } \\
\text { (3) Unacceptable risk for dangerousness to others as indicated by homicidal (or other violent) ideation, } \\
\text { intent or plan or action, or use of illegal weapons }\end{array}$} \\
\hline \multicolumn{3}{|c|}{$\begin{array}{l}\text { CDI: children's depression inventory; CDI-P: children's depression inventory-parent version; K-SADS-PL: Kiddie schedule for affective disorder } \\
\text { schizophrenia-present and lifetime version. } \\
\text { TABLE 2: Description of adolescent treatment sessions. }\end{array}$} \\
\hline Session & Week & Session content \\
\hline Session 1 & Week 1 & $\begin{array}{l}\text { Overview of the program, description of healthy eating and physical activity, and difference } \\
\text { between dieting and lifestyle change }\end{array}$ \\
\hline Session 2 & Week 2 & Logging food and movement, reading food labels, and avoiding food traps \\
\hline Session 3 & Week 3 & Managing emotions, avoiding sneak eating, and psychological versus physiological hunger \\
\hline Session 4 & Week 4 & Tools to increase health and wellness (e.g., Stoplight Eating Plan, Healthy Plate) \\
\hline Session 5 & Week 6 & $\begin{array}{l}\text { Staying motivated, increasing physical activity, everyday lifestyle movement, and decreasing } \\
\text { sedentary behavior }\end{array}$ \\
\hline Session 6 & Week 8 & Changing self-talk to be more positive, developing a healthy body image and self-esteem \\
\hline Session 7 & Week 10 & Being more self-aware with regards to eating, being active, and staying positive \\
\hline Session 8 & Week 12 & Overcoming barriers, planning ahead for healthy meals, special occasions, and eating out \\
\hline Booster Session 1 & Week 16 & Coping with PCOS \\
\hline Booster Session 2 & Week 20 & Planning for the future \\
\hline Booster Session 3 & Week 24 & Reflecting on the intervention \\
\hline
\end{tabular}

an average loss of $1.2( \pm .3)$ kilograms over the course of the intervention, with a promising effect size of .45. For additional information about the intervention, refer to Rofey et al. [23].

Overall, seven adolescent-parent dyads included in the present study did not have data at treatment completion. The sample that completed the intervention was younger $(M=15$ versus $M=16, p<.05)$ and had a lower BMI $(M=35$ versus $M=44, p<.05)$. Using an intent to treat analysis, there was a trend for greater change in BMI $(M=1.78$ versus $M=0.25, p=.087)$, and no differences on race $(t(39)=-.370 ; p=.714)$.

\subsection{Instruments}

2.3.1. General Information Sheet (GIS). The GIS has been used in previous studies and includes age, race, gender, religion, school placement, and SES using an occupationbased measure, the Revised Duncan (TSEI) [42, 43]. Contact information was requested, which may be used for locating subjects in the future.

2.3.2. Psychiatric Screener. Participant screening involved a two-step process. The first step was based on self-report on the Children's Depression Inventory (CDI) [44], given that this study was part of a larger intervention aimed to address the connection between mood and weight. The CDI consists of 27 items assessing depressive symptomatology; inter-item reliability: $(\alpha=.75)$. The total score on the CDI had to be at least 10 at recruitment [44]. This cutoff CDI score was chosen to be consistent with the usual cut-off scores for depressive symptoms in other physically ill populations [45-47]. The second step in the screening process was a semistructured psychiatric interview, the Kiddie Schedule for Affective Disorders and Schizophrenia-Present and Lifetime Version (K-SADS-PL) [38]. The K-SADS-PL is a semistructured interview designed to ascertain present episode and lifetime history of psychiatric illness according to DSM-IV criteria [48]. Inter-rater and test-re-test reliability have been established, as well as convergent and discriminant validity [49-51]. This measure was used to document the presence of specific pre- and post-treatment symptoms. Participants who endorsed symptoms commensurate with a diagnosis of minor or major depressive disorder were offered entry into the intervention portion of the study. Participants that were not eligible or chose not to participate were referred, if clinically appropriate, for the appropriate clinical services.

2.3.3. Weight Loss Behavior-Stage of Change Scale (WLBSOC) [52]. Adolescents and parents completed the WLBSOC [52] at baseline and intervention completion (six 
months). The WLB-SOC is a self-report measure that assesses SOC on the following five weight control domains: increasing dietary portion control, decreasing dietary fat, increasing fruit and vegetable consumption, increasing usual physical activity, and increasing planned exercise. Although there are two physical activity domains, increasing usual physical activity and increasing planned exercise, we use only the increasing usual physical activity domain in the current study, as it is validated [52]. The portion control, fruits and vegetables, and usual physical activity subscales have nine items, while the dietary fat subscale has 11 items. Reliabilities for the subscales on the adolescent measure were generally high and ranged from $\alpha=.70-.87$ at baseline and $\alpha=.75-.91$ at treatment completion. For the parent measure, reliabilities ranged from $\alpha=.67-.86$ at baseline and $\alpha=.70-.89$ at treatment completion. Participants responded to items that assess health behaviors they currently perform or plan to perform in the future, such as drinking a glass of water before meals or using low-fat salad dressing.

Participants responded to examples of behaviors within each domain using a 1-to-5 rating scale that corresponds to the five SOC. The response set ranged from $1=$ "I do not do this at least half the time now and I have no plans to do this" (precontemplation) to $5=$ "I do this at least half the time now and I have been doing this for more than 6 months" (maintenance), for example, in response to the item "Eat at least 5 servings of fruits and vegetables per day." An overall SOC score was then determined for each of the four weight control behaviors, using guidelines provided by Sutton and colleagues [52]. The overall SOC scores for each domain were then added together to provide a "total SOC" score, representing an individual's overall readiness to engage in behaviors related to weight loss. Higher "total SOC" scores represented greater readiness to engage in weight loss-related behaviors.

2.4. Data Analysis. For the portion control, dietary fat, fruits and vegetables, and usual physical activity domains, Chi Square analyses were conducted to provide the distribution of adolescents and parents in early SOC (precontemplation, contemplation, and preparation stages) versus later SOC (action and maintenance stages) at baseline and treatment completion (six months). Extant data indicate that there is a difference between those who express an intention to change a problem behavior in the near future (preparation) and individuals already in the process of making changes (action). Thus, we chose to divide the stages into "pre-action" (early SOC) and "action" (later SOC) groups [53]. Linear regression analyses were conducted using adolescent total SOC and parent total SOC as predictors of adolescent change in BMI from baseline to treatment completion (six months). Two sets of linear regression analyses were conducted with both adolescent total SOC and parent total SOC predictors. First, total baseline SOC (dietary portion control score + dietary fat score + fruits and vegetables score + usual physical activity score) for both adolescents and parents was analyzed as a predictor of adolescent change in BMI. Second, changes in adolescent and parent total SOC scores were used as predictors of change in adolescent BMI. Samples for Chi
Square $(n=31)$ and linear regression analyses $(n=40)$ differed due to missing adolescent and parent SOC data at treatment completion.

\section{Results}

Age, race, and BMI did not significantly differ between participants included in Chi Square analyses and those included in the linear regression analyses [age $(t(69)=$ $-.745 ; p=.459)$, race $(t(69)=.286 ; p=.776)$, BMI $(t(69)=-.567 ; p=.572)]$. Mean total baseline SOC for adolescents was $9.92(S D=2.73)$ and for parents was $11.79(S D=3.46)$, on a scale ranging from zero to twenty. Total SOC score is not indicative of a particular SOC (e.g., contemplation); it provides information that an individual is in some respects changing "more" or "less" in relation to others. Thus an increase in total SOC score suggests that a person is moving closer to a later SOC, such as the action stage or beyond. Mean total change in SOC from baseline to treatment completion for adolescents was $2.64(S D=2.64)$ and for parents was $2.04(S D=3.21)$. Paired samples $t$ tests indicated that both adolescents and parents significantly increased their SOC by an average of two points throughout the course of the intervention $(p<.001$ and $p=.005$, respectively). On average, adolescent BMI scores decreased by 0.52 $(S D=2.18)$ and BMI percentile decreased by $0.94(S D=$ 2.41) percentile units from baseline to treatment completion. Preliminary analyses revealed outliers for mean change in adolescent BMI from baseline to treatment completion, therefore data were analyzed both with and without outliers. Results for descriptive analyses are reported using the full sample (outliers included), as results without outliers were not significantly different. Primary analyses (Hypotheses 1 and 2) and exploratory analyses were conducted both with and without outliers. Results significantly differed across these analyses, therefore both sets of results are presented in Table 4. Mean total baseline SOC was $9.85(S D=2.88)$ for adolescents and $11.80(S D=3.62)$ for parents, on a scale ranging from zero to twenty. Mean total change in SOC from baseline to treatment completion for adolescents was 2.77 $(S D=2.69)$ and for parents was $2.48(S D=2.89)$. Paired samples $t$-tests indicated that both adolescents and parents significantly increased their SOC by an average of two points throughout the course of the intervention (for both groups, $p<.001)$. On average, adolescent BMI scores decreased by $0.17(S D=1.30)$ and BMI percentile decreased by 0.51 $(S D=1.36)$ percentile units from baseline to treatment completion. Age, race, and BMI did not significantly differ between outliers and nonoutliers: age $(t(38)=.426 ; p=$ $.672)$, race $(t(38)=1.27 ; p=.212)$, BMI $(t(38)=.081$; $p=.936)$.

The first aim of the current study was to examine SOC in adolescents and parents at the initiation of a weight loss intervention. The distribution of adolescents and parents in early versus later SOC at baseline is presented in Figure 1, which illustrates that the majority of adolescents and parents were in early SOC for all four weight control behaviors at baseline. 


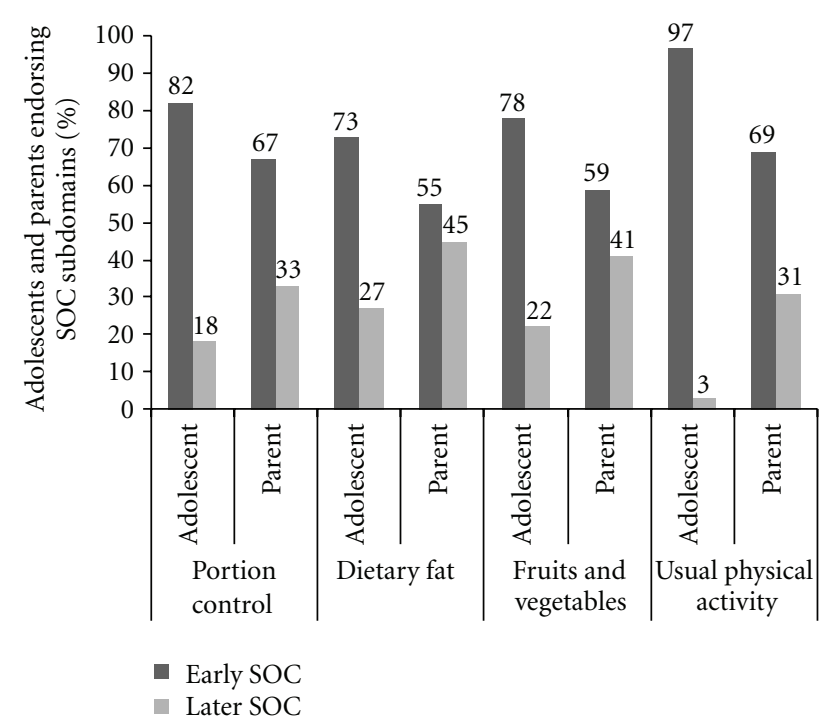

Figure 1: Distribution of Adolescents and Parents in Early SOC versus Later SOC at Baseline.

Figure 2 presents data on the distribution of adolescents and parents in early versus later SOC at treatment completion, following the six-month intervention. As indicated in Figure 2, the majority of adolescents were still in early SOC for increasing dietary portion control and increasing usual physical activity at treatment completion but endorsed later SOC for decreasing dietary fat and increasing fruit and vegetables. With regard to parent distributions in early versus later SOC at treatment completion, the majority of parents were in later SOC for the fruits and vegetables and usual physical activity domains, but not for the portion control or dietary fat domains (Figure 2).

3.1. Primary Analyses. Results shown in Table 3 indicate that adolescent total baseline SOC did not significantly predict change in adolescent BMI from baseline to treatment completion. Given our proposal that baseline SOC may not be the best predictor of change in BMI, it was equally important to look at change in SOC over time. Thus, we also examined change in total adolescent SOC from baseline to treatment completion as predictors of change in adolescent BMI. Results shown in Table 3 indicate that adolescent change in total SOC did not significantly predict adolescent change in BMI from baseline to treatment completion. Overall, the data presented in Table 3 do not support our hypothesis that adolescent baseline SOC scores would predict adolescent change in BMI from baseline to treatment completion (with outliers removed, there were no differences in results for adolescent predictors; see Table 3). Additionally, change in total adolescent SOC from baseline to treatment completion also did not predict change in adolescent BMI (Table 3).

Results shown in Table 4 indicate that parent total baseline SOC also did not significantly predict adolescent change in BMI from baseline to treatment completion. However, when we examined change in total parent SOC from baseline to treatment completion as a predictor of adolescent change in BMI, results (Table 4) indicated that change in parental

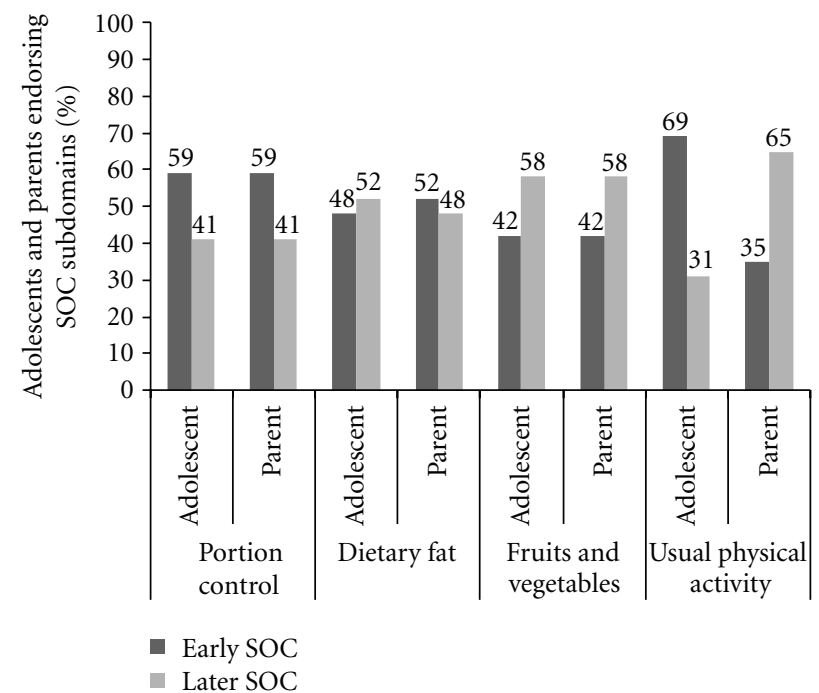

Figure 2: Distribution of Adolescents and Parents in Early SOC versus Later SOC at Treatment Completion.

total SOC reports from baseline to treatment completion significantly predicted adolescent change in BMI from baseline to treatment completion $\left(t(24)=2.15, p=0.043 ; \eta^{2}=\right.$ 0.42 ). To determine which weight control domains were driving this effect, we subsequently analyzed change in parent subscale SOC scores as predictors of adolescent change in BMI from baseline to treatment completion. As indicated in Table 4, a main effect of change in parent dietary fat SOC scores from baseline to treatment completion on change in adolescent BMI approached statistical significance $(t(26)=$ $\left.1.76, p=0.091 ; \eta^{2}=0.23\right)$. However, change in portion control SOC, fruits and vegetables SOC, and usual physical activity SOC did not significantly predict adolescents' change in BMI from baseline to treatment completion. With outliers removed, a main effect of change in parental total SOC reports from baseline to treatment completion on adolescent change in BMI from baseline to treatment completion approached statistical significance $(t(21)=1.81, p=0.087$; $\eta^{2}=0.53$ ); additionally, a main effect of change in parent SOC score for fruits and vegetables on adolescent change in BMI from baseline to treatment completion also approached statistical significance $\left(t(21)=1.95, p=0.066 ; \eta^{2}=0.32\right)$, see Table 4. Although the data presented in Table 4 did not support Hypothesis 2, exploratory analyses revealed that change in total parent SOC from baseline to treatment completion significantly predicted change in adolescent BMI from baseline to treatment completion. Additionally, a trend emerged for change in parent dietary fat SOC as a predictor of adolescent change in BMI. With outliers removed, trends emerged for change in parent total SOC and change in parent fruit and vegetable SOC as predictors of adolescent change in BMI from baseline to treatment completion.

\section{Discussion}

The key aim of the current investigation was to examine the relation between adolescents' and parents' self-reported readiness to change health-related behaviors and change in 
TABLE 3: Regression analyses for adolescent change in BMI by stage of change from baseline to treatment completion.

\begin{tabular}{lcccccrrr}
\hline \multirow{2}{*}{ Adolescent predictors } & \multicolumn{3}{c}{ Outliers included } & \multicolumn{4}{c}{ Outliers removed } \\
& $R^{2}$ & $B$ & Beta & $p$ & $R^{2}$ & $B$ & Beta & \\
\hline Main effect & & & & & & \\
Total baseline SOC & .014 & .093 & .117 & .483 & .013 & .051 & .116 \\
Total change in SOC & .000 & .008 & .013 & .947 & .016 & .054 & .126 \\
\hline
\end{tabular}

${ }^{*} p<0.05$; total baseline SOC: sum of SOC scores for four SOC subscales (portions + dietary fat + fruits and vegetables + physical activity); total change in SOC: difference of total baseline SOC and total SOC at treatment completion.

TABLE 4: Regression analyses for adolescent change in BMI by stage of change from baseline to treatment completion.

\begin{tabular}{|c|c|c|c|c|c|c|c|c|}
\hline \multirow{2}{*}{ Parent predictors } & \multicolumn{4}{|c|}{ Outliers included } & \multicolumn{4}{|c|}{ Outliers removed } \\
\hline & $R^{2}$ & $B$ & Beta & $p$ & $R^{2}$ & $B$ & Beta & $p$ \\
\hline \multicolumn{9}{|l|}{ Main effect } \\
\hline Total baseline SOC & .020 & .094 & .141 & .426 & .006 & .028 & .076 & .690 \\
\hline Total change in SOC & .173 & $.283^{*}$ & .416 & .043 & .147 & $.168^{\mathrm{t}}$ & .383 & .087 \\
\hline Change in portion control SOC & .001 & .061 & .039 & .851 & .005 & .063 & .072 & .746 \\
\hline Change in dietary fat SOC & .114 & $.483^{\mathrm{t}}$ & .338 & .091 & .063 & .242 & .250 & .249 \\
\hline Change in fruits and vegetables SOC & .114 & .695 & .337 & .107 & .166 & $.479^{t}$ & .408 & .066 \\
\hline Change in physical activity SOC & .081 & .369 & .285 & .177 & .035 & .139 & .188 & .414 \\
\hline
\end{tabular}

${ }^{*} p<0.05 ;{ }^{\mathrm{t}} p<0.10$; total baseline SOC: sum of SOC scores for four SOC subscales (portions + dietary fat + fruits and vegetables + physical activity); total change in SOC: difference of total baseline SOC and total SOC at treatment completion; change in (portions, dietary fat, fruits and vegetables, or physical activity) SOC: difference of SOC subscale at baseline and at treatment completion.

adolescent BMI during the course of a weight management intervention. Specifically, this study focused on adolescents and parents participating in a family-based weight management intervention for obese female adolescents with PCOS. Readiness to change was assessed using the SOC model [12]. Results revealed that the majority of adolescents and parents endorsed early SOC (versus later SOC) for all four weight control behaviors at baseline (increasing dietary portion control, decreasing dietary fat, increasing fruit and vegetable consumption, and increasing usual physical activity). Overall, these findings are consistent with evidence that individuals entering weight management programs have not yet implemented weight-related behavior changes consistent with being in a later SOC, for example, the action stage [54]. Prior to the end of the intervention, it is possible that adolescents and parents thought changes were important, but were not adequately confident in their abilities to complete these changes. At treatment completion, the majority of adolescents were still in early SOC for increasing portion control and increasing usual physical activity, compared to being in later SOC for decreasing dietary fat and increasing fruits and vegetables. Parents at treatment completion were still in early SOC for increasing portion control and decreasing dietary fat intake, while they endorsed later SOC for increasing fruit and vegetable intake and usual physical activity. Ultimately, both adolescents and parents endorsed later SOC for increasing fruit and vegetables and early SOC for increasing portion control. It is likely that increasing fruits and vegetables is relatively easy to change because it involves a specific dietary behavior, while increasing portion control may be difficult for both adolescents and parents as it may be a more comprehensive (and daunting) change that affects most dietary choices.
At treatment completion, adolescents and parents reported identical distributions in early versus later SOC for increasing portion control and increasing fruits and vegetables, with the majority of groups endorsing early SOC for portion control and later SOC for fruits and vegetables. Furthermore, roughly half of adolescents and parents endorsed later SOC for decreasing dietary fat (see Table 4) at treatment completion. However, the groups differed drastically in their readiness to change usual physical activity. The majority of adolescents were still in early SOC for increasing physical activity at treatment completion, while the majority of parents endorsed later SOC for this domain. It is possible that adolescents who are motivated to change dietary behaviors do not necessarily want to focus (or are ready to focus) on physical activity, which was indicated by our finding no correlation between adolescents' readiness to increase usual physical activity and their readiness to increase portion control or fruit and vegetable intake.

Considering adolescents' readiness to increase engagement in physical activity is separate from other weightrelated behaviors, it is necessary to highlight the finding that the majority of adolescents were still in early SOC for increasing usual physical activity at treatment completion. This may suggest that increasing usual physical activity is a particularly challenging lifestyle change for adolescents and potentially requires extra attention from interventionists. Extant data suggests that physical activity is the key predictor in weight loss, especially for adults who plateau in their weight loss and/or individuals attempting to maintain weight loss [55]. On the other hand, engagement in sedentary behaviors may have negatively impacted adolescents' readiness to increase physical activity. Research in children has shown that engagement in sedentary behaviors is one of the greatest barriers to engagement in physical 
activity [56, 57], perhaps because obese youth find sedentary behaviors more reinforcing [58]. Easy access to sedentary behaviors (e.g., computer, television, video games), limited access to opportunities to engage in physical activity (e.g., gyms, recreational facilities, parks), and comparatively low immediate reinforcing value of physical activity are potential reasons why obese adolescents find sedentary leisure-time activities more reinforcing, which consequently leads to weight gain. Previous work has indicated that reducing sedentary behaviors in children is associated with increases in physical activity that are similar to increases found when targeting physical activity directly [59-61]. Since the majority of adolescents in the present study were still in early SOC for increasing physical activity at treatment completion, future interventions may consider focusing on decreasing sedentary behaviors as a means to increase physical activity, instead of targeting physical activity alone. Additionally, this result supports the importance of curriculum-based physical activity in school as an avenue for providing adequate time for exercise in adolescents who may not be getting this outside of school.

Our hypothesis that adolescent baseline SOC scores would significantly predict adolescent change in BMI from baseline to treatment completion was not supported. Exploratory analyses examining the association between adolescent change in total SOC from baseline to treatment completion and change in adolescent BMI from baseline to treatment completion were also not significant. Given that this sample of adolescents had comorbid depressive symptoms consistent with a diagnosis of subclinical or clinical depression, they may have lacked sufficient motivation to make health-related changes. This is a salient factor to consider in a sample of obese adolescents whose depressive symptoms may inhibit their ability to experience significant weight loss and subsequent weight management. As stated above, there is a paucity of evidence regarding how depressive symptoms may affect adolescent motivation or SOC. Therefore, the present study helps to inform future research targeting female adolescents with physical and psychiatric diagnoses and may ultimately inform standard treatment of this population. While depression typically negatively affects motivational factors, our sample of adolescents with PCOS has preliminarily shown that their depressive symptoms may be weight driven and, therefore, may be a more positive motivational factor, such that these adolescents may feel more positive if they are able to succeed with weight loss $[62,63]$.

Our hypothesis that parent baseline SOC score would be a stronger predictor of adolescent change in BMI from baseline to treatment completion was not upheld. However, exploratory analyses revealed that change in parent total SOC from baseline to treatment completion did significantly predict change in adolescent BMI from baseline to treatment completion. It is likely that both adolescents and parents are less ready to make weight control changes prior to starting an intervention because they are less aware of important weight control behaviors and how they can incorporate them into their lives. Thus the change in total SOC variable may reflect the impact of the intervention itself on change in BMI and be a better predictor of subsequent adolescent change in BMI than total SOC at baseline. This finding also highlights the importance of examining change in SOC throughout the course of a weight management intervention, as well as the importance of using client-centered approaches.

Overall, the finding that parent change in total SOC from baseline to treatment completion significantly predicts adolescent change in BMI from baseline to treatment completion is consistent with previous evidence for the importance of parent involvement in and commitment to child weight management interventions [37]. Although this significant finding became a trend after the removal of the four outliers, it is possible that in a larger sample the significant association could be upheld. In support of how powerful parental influence may be for weight management in children, Golan and colleagues $[64,65]$ found that targeting the parent as the primary agent of child weight loss compared to targeting children as the primary agent resulted in significantly more child weight loss at program termination and at 1-, 2-, and 7-year follow-up. Similarly, a review by Epstein et al. [66] presented support for family-based programs for both shortand long-term weight loss among youth. Consequently, there is demonstrated support for the role of family-based treatment in child weight management, yet there has been very little work with regard to the role of parents in treatment for adolescents. Although parents generally have less influence on adolescent behavior, as compared to child behavior, the present findings illuminate the possibility that parent involvement does play a salient role in the success of weight management interventions for adolescents. Thus these results indicate the necessity for further research into the parent-adolescent dyad and more specifically into the impact of parent readiness to make weight-related changes on the effectiveness of adolescent weight management.

Parent change in readiness to decrease dietary fat intake and to increase fruit and vegetable intake from baseline to treatment completion emerged as specific weight control behaviors related to change in adolescent BMI. These trends may be indicative of true associations and could potentially be substantiated if tested in a larger sample. Since parents making changes in one specific weight control domain translated to adolescent weight change, it is likely important for parent-interventionist sessions to involve discussing specific, succinct behavioral changes and setting goals $[64,65]$.

4.1. Clinical Implications. The importance of parent involvement in adolescents' weight management is potentially important to future pediatric weight management interventions. Our results highlight the salient role parents play in adolescents' weight management and may even suggest that future interventions emphasize using MI principles with parents, or at the very least, a parent-only group $[29,67$, 68]. Because MI is a client-centered therapeutic approach that aims to meet persons at their current SOC and help solve ambivalence surrounding making a behavior change [39], it may serve as a particularly useful technique for enhancing readiness to change. Its directive, nonjudgmental approach allows interventionists to meet adolescents in their particular stage and work through their ambivalence to move 
to later SOC. Furthermore, MI allows for the possibility of movement back and forth between different stages, thereby alleviating any pressure related to a fear of failure. Future interventions that implement assessment of SOC with an MI spirit are strongly suggested over psychoeducational programs. Psychoeducation may lead to overestimating an individual's readiness to change, and MI may increase the likelihood of parents serving as a supportive coach for their adolescents as they instill family-based changes in the household. Furthermore, using MI techniques with parents may advance their readiness to make positive weight-related changes, which may directly influence their adolescent's readiness to also make changes. Assessment of parent and adolescent SOC allows for tailored interventions that are congruent with participants' individual stages of readiness, which can in turn improve treatment outcomes [54].

4.2. Study Limitations and Future Directions. This investigation was subject to a number of limitations. First, this study specifically focused on obese adolescent females with PCOS and comorbid depression. Thus, it is possible that these results are not generalizable to other populations, particularly obese male adolescents or adolescents without cooccuring physical or psychiatric diagnoses. The use of selfreported questionnaire data may have increased response bias motivated by social desirability factors. This study did not investigate parent BMI or parent change in BMI as predictors of adolescent change in BMI, which may be important factors in adolescents' readiness to make weightrelated behavior changes and in change in BMI. Additionally, while using BMI z-score is generally recommended for ages $2-19$, our sample ranged in age from 13 to 20 years therefore we chose to use change in BMI as our outcome measure. Furthermore, it is unclear if the parents' readiness to change reflected motivation to make weight-related changes for themselves or possibly their motivation to make changes on behalf of their daughters. It would be valuable to elucidate which aspect of parents' motivation is more predictive of adolescent change in BMI, to better inform and structure parent involvement in treatment. It is also possible that the depression experienced by the adolescents and/or their medical diagnosis of PCOS may have influenced their motivation to make weight-related changes. Future research may resolve these questions by examining the following: first, if depression is related to adolescents' readiness to make health changes; second, the directionality of that relationship; third, how the presence of a medical diagnosis is related to readiness to make changes. Finally, although SOC was shown to predict change in adolescent BMI, the present study did not indicate SOC as a predictor of actual behavior (e.g., portion control SOC as a predictor of actual changes in portion control behavior). Although it is likely that, individually, each SOC subscale is more related to readiness to engage in the actual behavior (e.g., decreasing dietary fat) than overall change in BMI, it is important to note that weight control involves a complete lifestyle change. This supports the use of examining the four subscales together as total SOC, as a composite score of overall readiness to change one's lifestyle, which may better predict subsequent change in BMI.

Study results lend to suggestions for future research directions. First, it may be important to evaluate adolescent and parent SOC at multiple time points throughout a pediatric obesity intervention, possibly to tailor the progress of the intervention to focus more directly on SOC subscales (e.g., physical activity) that adolescents and parents are still not yet ready to change. Second, given that parent SOC significantly changed from baseline to treatment completion and adolescent SOC did not, future investigations might focus on first increasing parent readiness to change as a means to subsequently increase adolescent readiness to change. It may also be informative to examine how techniques such as MI could improve patient and/or parent SOC with regard to behaviors necessary for successful weight management or if SOC could be useful in predicting the effectiveness of MI compliant therapists in initiating change. Finally, future studies may examine the correlation between depressive symptoms (using both self-report and clinical interview assessments) and SOC scores, to determine how depression influences readiness to change weight control behaviors, particularly adolescents' motivation and perceived self-efficacy for making broad weight-related changes.

Despite the aforementioned limitations, our results that parent readiness to change plays an integral role in adolescent weight loss substantially contribute to the literature. Future studies should consider broadening the study sample to include boys, assessing parent and adolescent SOC at multiple time points and examining the role adolescent depression plays in motivation for lifestyle change. Treatment programs will potentially benefit from implementing assessment of SOC and MI. Overall, parent readiness to change weightrelated behaviors is more predictive of adolescent change in BMI than adolescent readiness to change. These findings have important implications for future research and possibly prevention and treatment efforts for obese adolescents with co-occurring physical illness and depression.

\section{Conflicts of Interest}

The authors declare that they have no Conflicts of Interest.

\section{Acknowledgments}

These data are supported by K12-HD043441 (DLR) and K23HD061598 (DLR). The authors wish to acknowledge the Polycystic Ovary Syndrome Center of Children's Hospital of Pittsburgh, as well as Marguerite O'Hara, M. A. and Ronette Blake, M. S., for their assistance with this research project, including data management.

\section{References}

[1] C. L. Ogden, M. D. Carroll, L. R. Curtin, M. M. Lamb, and K. M. Flegal, "Prevalence of high body mass index in US children and adolescents, 2007-2008," Journal of the American Medical Association, vol. 303, no. 3, pp. 242-249, 2010. 
[2] R. Azziz, K. S. Woods, R. Reyna, T. J. Key, E. S. Knochenhauer, and B. O. Yildiz, "The prevalence and features of the polycystic ovary syndrome in an unselected population," Journal of Clinical Endocrinology and Metabolism, vol. 89, no. 6, pp. 2745-2749, 2004.

[3] B. O. Yildiz, E. S. Knochenhauer, and R. Azziz, "Impact of obesity on the risk for polycystic ovary syndrome," Journal of Clinical Endocrinology and Metabolism, vol. 93, no. 1, pp. 162168, 2008.

[4] S. Arslanian and S. Witchel, "Premature pubarche, insulin resistance, and adolescentpolycystic ovary syndrome," in Polycystic Ovary Syndrome, R. Chang, J. Heindel, and A. Dunaif, Eds., pp. 37-53, Marcel Dekker, New York, NY, USA, 2002.

[5] W. H. Dietz, "Overweight in childhood and adolescence," New England Journal of Medicine, vol. 350, no. 9, pp. 855-857, 2004.

[6] B. E. Saelens, J. F. Sallis, D. E. Wilfley, K. Patrick, J. A. Cella, and R. Buchta, "Behavioral weight control for overweight adolescents initiated in primary care," Obesity Research, vol. 10, no. 1, pp. 22-32, 2002.

[7] E. Hollinrake, A. Abreu, M. Maifeld, B. J. Van Voorhis, and A. Dokras, "Increased risk of depressive disorders in women with polycystic ovary syndrome," Fertility and Sterility, vol. 87, no. 6, pp. 1369-1376, 2007.

[8] D. L. Rofey, R. B. Noll, V. Weisbrod, K. B. Van Schaick, E. Szigethy, and S. Arslanian, "Examining the etiology of depression in adolescents with Polycystic Ovary Syndrome (PCOS)," in Presentation at the Society of Pediatric Psychology, Cincinnati, Ohio, April 2007.

[9] M. J. Himelein and S. S. Thatcher, "Depression and body image among women with polycystic ovary syndrome," Journal of Health Psychology, vol. 11, no. 4, pp. 613-625, 2006.

[10] S. Elsenbruch, S. Hahn, D. Kowalsky et al., "Quality of life, psychosocial well-being, and sexual satisfaction in women with polycystic ovary syndrome," Journal of Clinical Endocrinology and Metabolism, vol. 88, no. 12, pp. 5801-5807, 2003.

[11] N. L. Rasgon, R. C. Rao, S. Hwang et al., "Depression in women with polycystic ovary syndrome: clinical and biochemical correlates," Journal of Affective Disorders, vol. 74, no. 3, pp. 299-304, 2003.

[12] C. C. DiClemente and J. O. Prochaska, "Self-change and therapy change of smoking behavior: a comparison of processes of change in cessation and maintenance," Addictive Behaviors, vol. 7, no. 2, pp. 133-142, 1982.

[13] A. Steptoe, S. Wijetunge, S. Doherty, and J. Wardle, "Stages of change for dietary fat reduction: associations with food intake, decisional balance and motives for food choice," Health Education Journal, vol. 55, no. 1, pp. 108-122, 1996.

[14] K. A. Peterson and M. Hugbes, "Readiness to change and clinical success in a diabetes educational program," Journal of the American Board of Family Practice, vol. 15, no. 4, pp. 266$271,2002$.

[15] K. Glanz, R. E. Patterson, A. R. Kristal et al., "Stages of change in adopting healthy diets: fat, fiber, and correlates of nutrient intake," Health Education Quarterly, vol. 21, no. 4, pp. 499$519,1994$.

[16] G. W. Greene, N. Fey-Yensan, C. Padula, S. Rossi, J. S. Rossi, and P. G. Clark, "Differences in psychosocial variables by stage of change for fruits and vegetables in older adults," Journal of the American Dietetic Association, vol. 104, no. 8, pp. 12361243, 2004.

[17] N. L. Fahrenwald and S. N. Walker, "Application of the Transtheoretical Model of Behavior Change to the Physical Activity Behavior of WIC Mothers," Public Health Nursing, vol. 20, no. 4, pp. 307-317, 2003.
[18] A. L. Marshall, A. A. Bauman, N. Owen, M. L. Booth, D. Crawford, and B. H. Marcus, "Population-based randomized controlled trial of a stage-targeted physical activity intervention," Annals of Behavioral Medicine, vol. 25, no. 3, pp. 194202, 2003.

[19] B. H. Marcus and B. A. Lewis, "Physical activity and the stages of motivational readiness for change model," Research Digest, series 4, no. 1, 2003.

[20] B. H. Marcus and N. Owen, "Motivational readiness, selfefficacy and decision-making for exercise," Journal of Applied Social Psychology, vol. 22, pp. 3-16, 1992.

[21] S. J. Marshall and S. J. H. Biddle, "The transtheoretical model of behavior change: a meta-analysis of applications to physical activity and exercise," Annals of Behavioral Medicine, vol. 23, no. 4, pp. 229-246, 2001.

[22] M. G. Myers and S. A. Brown, "A controlled study of a cigarette smoking cessation intervention for adolescents in substance abuse treatment," Psychology of Addictive Behaviors, vol. 19, no. 2, pp. 230-233, 2005.

[23] D. L. Rofey, E. M. Szigethy, R. B. Noll, R. E. Dahl, E. Lobst, and S. A. Arslanian, "Cognitive-behavioral therapy for physical and emotional disturbances in adolescents with polycystic ovary syndrome: a pilot study," Journal of Pediatric Psychology, vol. 34, no. 2, pp. 156-163, 2009.

[24] D. S. Kirschenbaum, J. N. Germann, and B. H. Rich, "Treatment of morbid obesity in low-income adolescents: effects of parental self-monitoring," Obesity Research, vol. 13, no. 9, pp. 1527-1529, 2005.

[25] K. Resnicow, R. Taylor, M. Baskin, and F. McCarty, "Results of go girls: a weight control program for overweight AfricanAmerican adolescent females," Obesity Research, vol. 13, no. 10, pp. 1739-1748, 2005.

[26] K. Resnicow, A. L. Yaroch, A. Davis et al., "GO GIRLS!: development of a community-based nutrition and physical activity program for overweight African-American adolescent females," Health Education \& Behavior, vol. 27, pp. 616-631, 2000.

[27] T. Wysocki, M. A. Harris, L. M. Buckloh et al., "Effects of behavioral family systems therapy for diabetes on adolescents' family relationships, treatment adherence, and metabolic control," Journal of Pediatric Psychology, vol. 31, no. 9, pp. 928938, 2006.

[28] S. Channon, V. J. Smith, and J. W. Gregory, "A pilot study of motivational interviewing in adolescents with diabetes," Archives of Disease in Childhood, vol. 88, no. 8, pp. 680-683, 2003.

[29] S. M. Berg-Smith, V. J. Stevens, K. M. Brown et al., "A brief motivational intervention to improve dietary adherence in adolescents," Health Education Research, vol. 14, no. 3, pp. 399-410, 1999.

[30] M. M. Black, E. R. Hager, K. Le et al., "Challenge! Health promotion/obesity prevention mentorship model among urban, black adolescents," Pediatrics, vol. 126, pp. 280-288, 2010.

[31] M. Golan and S. Crow, "Parents are key players in the prevention and treatment of weight-related problems," Nutrition Reviews, vol. 62, no. 1, pp. 39-50, 2004.

[32] L. L. Moore, D. A. Lombardi, M. J. White, J. L. Campbell, S. A. Oliveria, and R. C. Ellison, "Influence of parent's physical activity levels on activity levels of young children," Journal of Pediatrics, vol. 118, no. 2, pp. 215-219, 1991.

[33] J. O. Fisher, D. C. Mitchell, H. Smiciklas-Wright, and L. L. Birch, "Parental influences on young girls' fruit and vegetable, micronutrient, and fat intakes," Journal of the American Dietetic Association, vol. 102, no. 1, pp. 58-64, 2002. 
[34] K. E. Rhee, C. W. DeLago, T. Arscott-Mills, S. D. Mehta, and R. K. Davis, "Factors associated with parental readiness to make changes for overweight children," Pediatrics, vol. 116, no. 1, pp. e94-e101, 2005.

[35] A. J. Stunkard, M. S. Faith, and K. C. Allison, "Depression and obesity," Biological Psychiatry, vol. 54, no. 3, pp. 330-337, 2003.

[36] M. H. Zeller and A. C. Modi, "Predictors of health-related quality of life in obese youth," Obesity, vol. 14, no. 1, pp. 122130, 2006.

[37] S. E. Barlow, "Expert committee recommendations regarding the prevention, assessment, and treatment of child and adolescent overweight and obesity: summary report," Pediatrics, vol. 120, pp. S164-S192, 2007.

[38] J. Kaufman, B. Birmaher, D. Brent et al., "Schedule for affective disorders and schizophrenia for school-age childrenpresent and lifetime version (K-SADS-PL): initial reliability and validity data," Journal of the American Academy of Child and Adolescent Psychiatry, vol. 36, no. 7, pp. 980-988, 1997.

[39] W. Miller and S. Rollnick, Motivational Interviewing: Preparing People for Change, Guilford Press, New York, NY, USA, 2002.

[40] K. Resnicow, R. Davis, and S. Rollnick, "Motivational interviewing for pediatric obesity: conceptual issues and evidence review," Journal of the American Dietetic Association, vol. 106, no. 12, pp. 2024-2033, 2006.

[41] P. Teixeira, M. Silva, J. Mata, A. Palmeira, and D. Markland, "Motivation, self-determination, and long-term weight control," International Journal of Behavioral Nutrition and Physical Activity, vol. 9, article 1, 2012.

[42] K. Nakao and J. Treas, "The 1989 socioeconomic index of occupations: constructionfrom the 1989 occupational prestige scores," General Social Survey Methodological Report no. 74, University of Chicago, National Opinion Research Center, Chicago, Ill, USA, 1992.

[43] R. M. Hauser, "Measuring socioeconomic status in studies of child development," Child development, vol. 65, no. 6, pp. 1541-1545, 1994.

[44] M. Kovacs, Children's Depression Inventory Manual, MultiHealth Systems, North Tonawanda, NY, USA, 1992.

[45] E. Szigethy, S. W. Whitton, A. Levy-Warren, D. R. DeMaso, J. Weisz, and W. R. Beardslee, "Cognitive-behavioral therapy for depression in adolescents with inflammatory bowel disease: a pilot study," Journal of the American Academy of Child and Adolescent Psychiatry, vol. 43, no. 12, pp. 1469-1477, 2004.

[46] E. Szigethy, "Therapy for the brain and gut," Journal of Psychiatric Practice, vol. 11, no. 1, pp. 51-53, 2005.

[47] E. Szigethy, J. Carpenter, E. Baum et al., "Case study: longitudinal treatment of adolescents with depression and inflammatory bowel disease," Journal of the American Academy of Child and Adolescent Psychiatry, vol. 45, no. 4, pp. 396-400, 2006.

[48] American Psychiatric Association, Diagnostic and Statistical Manual of Mental, Washington, DC, USA, 4th edition, 1994.

[49] W. J. Chambers, J. Puig-Antich, and M. Hirsch, "The assessment of affective disorders in children and adolescents by semistructured interview. Test-retest reliability of the Schedule for Affective Disorders and Schizophrenia for school-age children," Archives of General Psychiatry, vol. 42, no. 7, pp. 696-702, 1985.

[50] J. Puig-Antich, H. Orvalschel, M. A. Tabrizi, and W. Chambers, The Schedule for Affective Disorders and Schizophrenia for School-Age Children-Epidemiologic Version, New York State
Psychiatric Institution and Yale University School of Medicine, New York, NY, USA, 1980.

[51] G. D. Gammon, K. John, and E. D. Rothblum, "Use of a structured diagnostic interview to identify bipolar disorder in adolescent inpatients: frequency and manifestations of the disorder," American Journal of Psychiatry, vol. 140, no. 5, pp. 543-547, 1983.

[52] K. Sutton, E. Logue, D. Jarjoura, K. Baughman, W. Smucker, and C. Capers, "Assessing dietary and exercise stage of change to optimize weight loss interventions," Obesity Research, vol. 11, no. 5, pp. 641-652, 2003.

[53] C. C. DiClemente, J. O. Prochaska, S. K. Fairhurst, W. F. Velicer, M. M. Velasquez, and J. S. Rossi, "The process of smoking cessation: an analysis of precontemplation, contemplation, and preparation stages of change," Journal of Consulting and Clinical Psychology, vol. 59, no. 2, pp. 295-304, 1991.

[54] J. O. Prochaska, C. C. DiClemente, and J. C. Norcross, "In search of how people change: applications to addictive behaviors," American Psychologist, vol. 47, no. 9, pp. 11021114, 1992.

[55] M. L. Klem, R. R. Wing, M. T. McGuire, H. M. Seagle, and J. O. Hill, "A descriptive study of individuals successful at long-term maintenance of substantial weight loss," American Journal of Clinical Nutrition, vol. 66, no. 2, pp. 239-246, 1997.

[56] C. J. Crespo, E. Smit, R. P. Troiano, S. J. Bartlett, C. A. Macera, and R. E. Andersen, "Television watching, energy intake, and obesity in US children: results from the Third National Health and Nutrition Examination Survey, 1988-1994," Archives of Pediatrics and Adolescent Medicine, vol. 155, no. 3, pp. 360365, 2001.

[57] W. H. Dietz and S. L. Gortmaker, "Do we fatten our children at the television set? Obesity and television viewing in children and adolescents," Pediatrics, vol. 75, no. 5, pp. 807-812, 1985.

[58] L. H. Epstein, J. A. Smith, L. S. Vara, and J. S. Rodefer, "Behavioral economic analysis of activity choice in obese children," Health Psychology, vol. 10, no. 5, pp. 311-316, 1991.

[59] L. H. Epstein, R. A. Paluch, C. C. Gordy, and J. Dorn, "Decreasing sedentary behaviors in treating pediatric obesity," Archives of Pediatrics and Adolescent Medicine, vol. 154, no. 3, pp. 220-226, 2000.

[60] L. H. Epstein, B. E. Saelens, M. D. Myers, and D. Vito, "Effects of decreasing sedentary behaviors on activity choice in obese children," Health Psychology, vol. 16, no. 2, pp. 107-113, 1997.

[61] L. H. Epstein, A. M. Valoski, L. S. Vara et al., "Effects of decreasing sedentary behavior and increasing activity on weight change in obese children," Health Psychology, vol. 14, no. 2, pp. 109-115, 1995.

[62] K. P. Jakubowski, J. S. Silk, R. Blake, R. E. Dahl, and D. L. Rofey, "Affective experience in the natural environments of female adolescents with primary depression vs. obesity and comorbid depression," Poster to be presented at the Society for Research on Adolescence Biennial Meeting, Vancouver, Canada, 2012.

[63] D. L. Rofey, J. J. Black, K. Olson, and E. Szigethy, "Novel type of depression in overweight youth," Poster presented at the annual meeting of the Obesity Society, San Diego, Cali, USA, 2010.

[64] M. Golan, A. Weizman, A. Apter, and M. Fainaru, "Parents as the exclusive agents of change in the treatment of childhood obesity," American Journal of Clinical Nutrition, vol. 67, no. 6, pp. 1130-1135, 1998.

[65] M. Golan and S. Crow, "Targeting parents exclusively in the treatment of childhood obesity: long-term results," Obesity Research, vol. 12, no. 2, pp. 357-361, 2004. 
[66] L. H. Epstein, R. A. Paluch, J. N. Roemmich, and M. D. Beecher, "Family-based obesity treatment, then and now: twenty-five years of pediatric obesity treatment," Health Psychology, vol. 26, no. 4, pp. 381-391, 2007.

[67] M. Irby, S. Kaplan, D. Garner-Edwards, S. Kolbash, and J. A. Skelton, "Motivational interviewing in a family-based pediatric obesity program: a case study," Families, Systems and Health, vol. 28, no. 3, pp. 236-246, 2010.

[68] K. Resnicow, F. McCarty, and T. Baranowski, "Are precontemplators less likely to change their dietary behavior? A prospective analysis," Health Education Research, vol. 18, no. 6, pp. 693-705, 2003. 


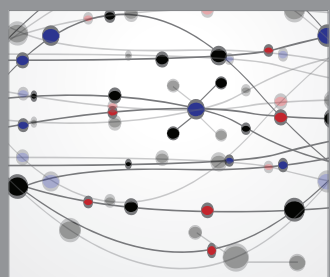

The Scientific World Journal
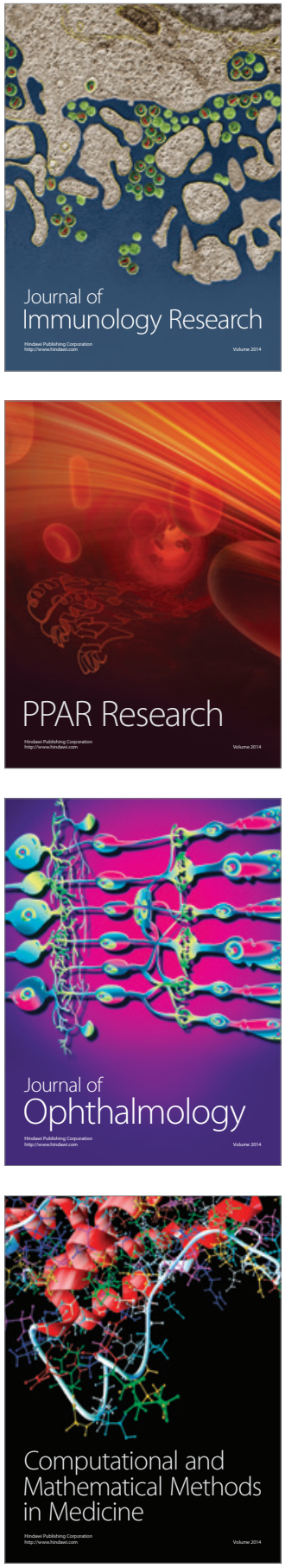

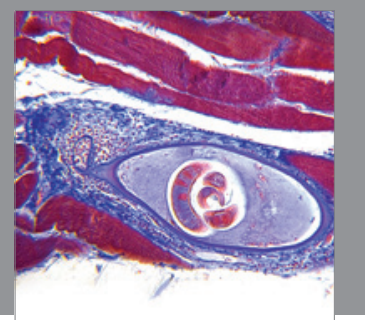

Gastroenterology

Research and Practice
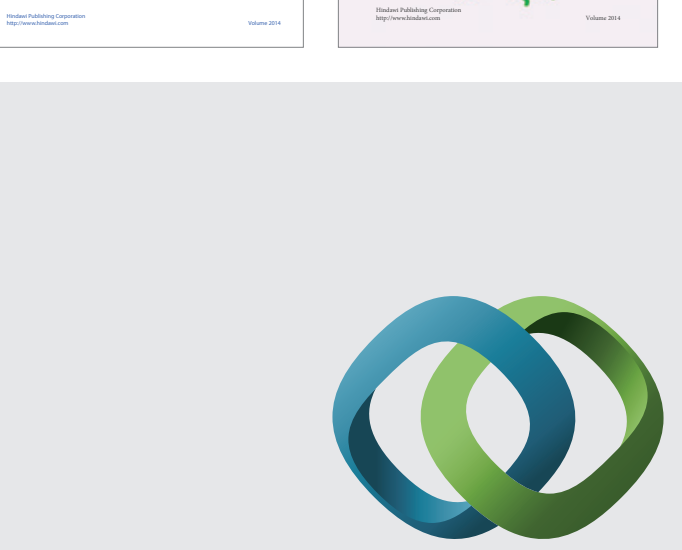

\section{Hindawi}

Submit your manuscripts at

http://www.hindawi.com
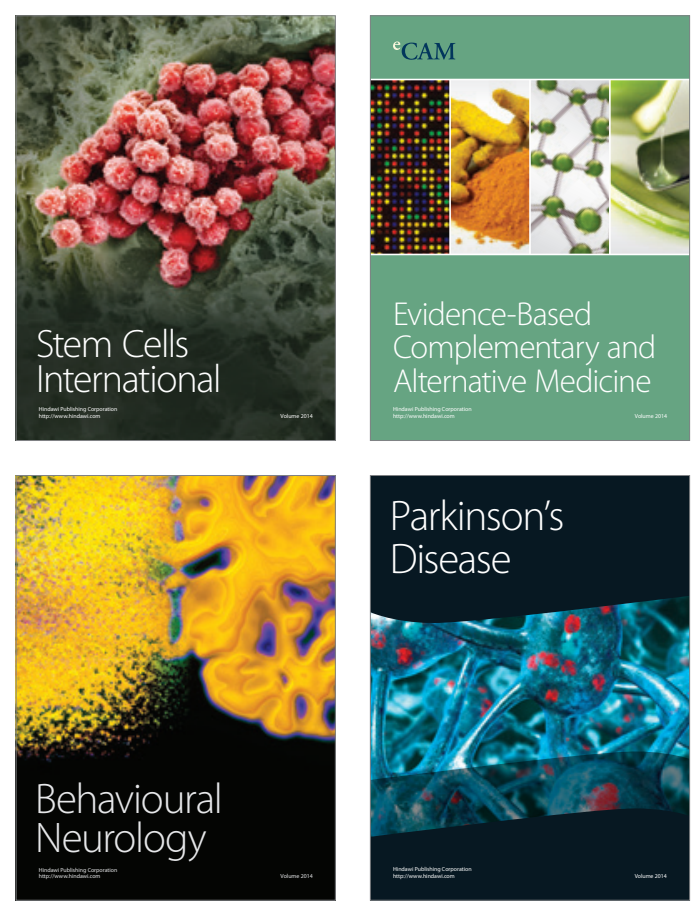

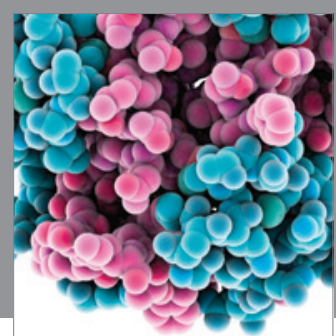

Journal of
Diabetes Research

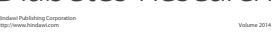

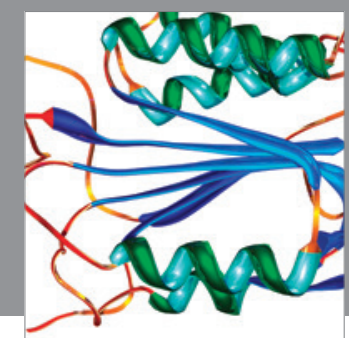

Disease Markers
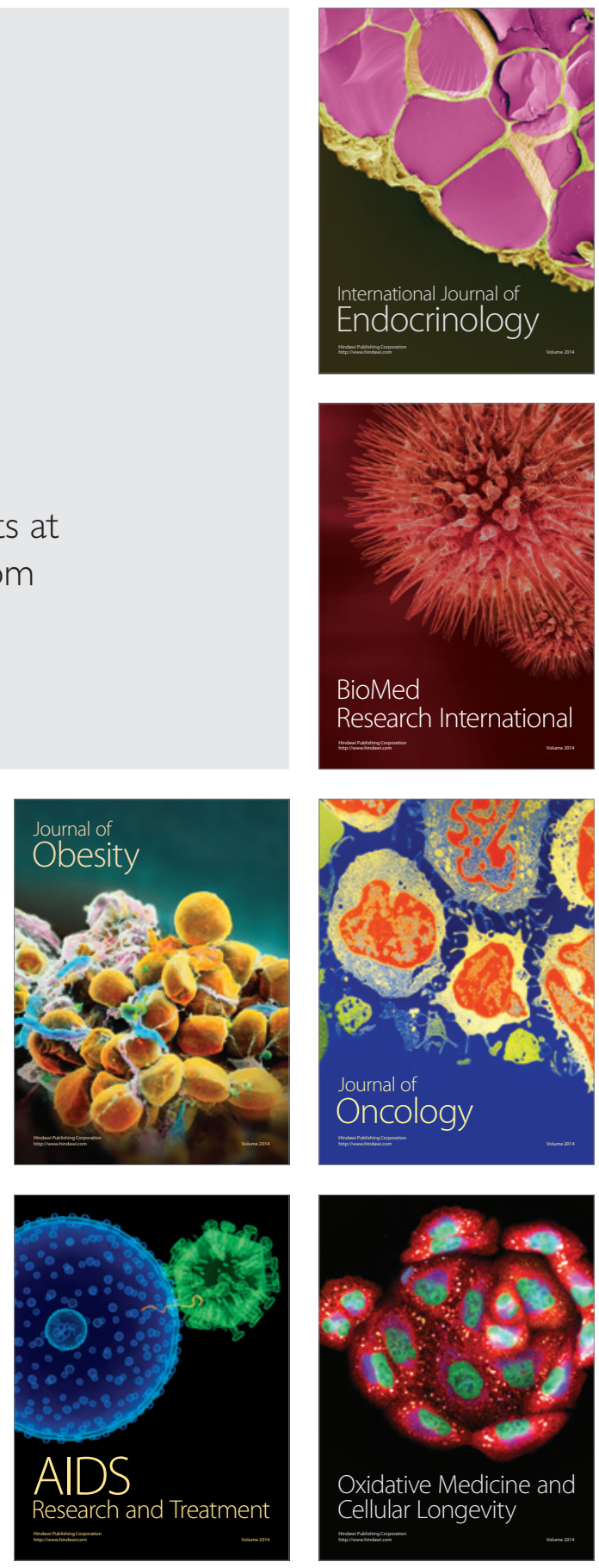